\title{
Production d'impulsions ultracourtes de rayons $X$ par interaction laser matière
}

\author{
J.P. Geindre, P. Audebert, A. Rousse et J.C. Gauthier \\ LULI, Ecole Polytechnique, 91128 Palaiseau cedex, France
}

\begin{abstract}
Résumé: Dans une première partie nous explicitons les caractères spécifiques qui gouvernent la physique de l'interaction laser matière en impulsion ultracourte. Les ordres de grandeur associés au champ de l'onde et a son interaction avec un électron sont donnés. L'importance de l'état initial de la cible sur l'absorption de l'énergie laser est explicitée, ainsi que ses conséquences sur l'intensité et la durée des émissions $X$. Nous montrons l'importance des effets non linaires sur la génération d'électrons suprathermiques durant l'interaction. Dans une seconde partie, la production de rayonnement $K_{\alpha}$ (obtenue par ionisation en couche interne) est démontrée dans nos conditions expérimentales. La fonction de distribution des électrons rapides responsable de cette ionisation est mesurée. En partant de ces données, nous qualifions ce type de plasma comme source intense et ultracourte pour des énergies comprises entre 1 et $10 \mathrm{keV}$.
\end{abstract}

\section{INTRODUCTION}

La génération d'impulsions $X$ ultracourtes et intenses est un challenge pour l'étude de la physique des plasmas de hautes températures et de hautes densités [1]. De tels plasmas sont produits quand une impulsion laser ultracourte et intense (100 fs, $>10^{16} \mathrm{~W} / \mathrm{cm}^{2}$ ) est focalisée sur des cibles solides [2-8].

L'étude de la dynamique temporelle des changements de phase des matériaux, ainsi que celle des changements de conformation des molécules biologiques [9-10] est l'une des applications les plus prometteuses de ce flash $\mathrm{X}$ incohérent et intense.

\subsection{Ordres de grandeur}

Avant d'aller plus loin, il est utile de rappeler les ordres de grandeur qui caractérisent un champ électromagnétique intense.

Dans le vide, une impulsion laser de haute intensité est caractérisé par une densité d'énergie électromagnétique considérable. Au delà de $3,410^{16}\left(\mathrm{~W} / \mathrm{cm}^{2}\right)$, le champ électrique de l'onde laser devient supérieur au champ atomique de l'atome d'hydrogène $\left(510^{9} \mathrm{~V} / \mathrm{cm}\right)$. 
Dans un plasma, il faut considérer l'oscillation des électrons dans le champ électrique du laser. L'amplitude de cette dernière devient une fraction non négligeable de la longueur d'onde du laser et la vitesse instantanée s'approche de celle de la lumière. L'énergie cinétique de l'électron oscillant devient supérieure à l'énergie thermique du plasma chaud. La pression associée à cette énergie devient donc supérieure à la pression cinétique du plasma.

A titre d'illustration le tableau ci-dessous présente l'évolution de ces grandeurs pour des flux allant de $210^{16}$ à $10^{18} \mathrm{~W} / \mathrm{cm}^{2}$ en polarisation circulaire et pour une longueur d'onde de $0.620 \mu \mathrm{m}$.

\begin{tabular}{|c|c|c|c|}
\hline Flux laser & $210^{16}\left(\mathrm{~W} / \mathrm{cm}^{2}\right)$ & $10^{17}\left(\mathrm{~W} / \mathrm{cm}^{2}\right)$ & $10^{18}\left(\mathrm{~W} / \mathrm{cm}^{2}\right)$ \\
\hline Elaser $_{\text {lV/cm) }}$ & $3,910^{9}$ & $910^{9}$ & $2.7510^{10}$ \\
\hline Densité EM $\left(\mathbf{J} / \mathbf{c m}^{3}\right)$ & $0,6610^{6}$ & $3,310^{6}$ & $3310^{6}$ \\
\hline Amplitude $(\AA)$ & 76 & 170 & 523 \\
\hline Vitesse (m/s) & $1.5710^{7}$ & $3.510^{7}$ & $1.110^{8}$ \\
\hline Énergie (keV) & 1.4 & 7.1 & 71 \\
\hline
\end{tabular}

\subsection{Interaction d'une impulsion laser ultracourte avec un solide}

Pendant la durée de l'impulsion laser ultracourte, l'expansion du plasma est faible. L'énergie laser peut donc être absorbée dans l'épaisseur de peau $[9,11]$ ce qui génère un plasma thermique de température égale à plusieurs centaines d'eV dont la densité est proche de la densité du solide. Ce plasma chaud émet un rayonnement $\mathrm{X}$ dont la durée est contrôlée par sa vitesse de refroidissement. Deux mécanismes y contribuent: la conduction thermique vers le solide froid et la détente adiabatique du plasma dans le vide. L'efficacité de ces deux mécanismes conduit à une émission $X$ dont la durée peut être proche de celle de l'impulsion laser .

Durant l'interaction, des électrons rapides sont directement générés par le champ électrique du laser [14-17]. Ceux-ci pénètrent en profondeur dans la cible et ejectent des électrons liés de la couche $\mathrm{K}$ des atomes ou des ions. Ce processus produit une émission $X$ lors de la décroissance radiative du système atomique (raie $\mathrm{K}_{\alpha}$ ) [7, 18-20]. Si le temps de vie des électrons rapides est inférieur à la durée de l'impulsion laser, la durée de l'émission $X$ sera pratiquement égale à cette dernière. Pour obtenir ce résultat, il faut que 1 'énergie des électrons ne soit pas trop élevée (typiquement moins de $20 \mathrm{keV}$ pour des cibles d'aluminium).

Afin d'analyser l'émission $K_{\alpha}$ générée durant l'interaction il faut étudier les mécanismes d'absorption non linéaires de l'énergie laser qui conduisent à la génération d'électrons suprathermiques.

\subsection{La conversion en électrons rapides.}

\subsubsection{Théorie}

Un électron oscillant dans le champ d'une onde laser n'absorbe pas d'énergie, il se contente de l'échanger de façon réversible avec le champ électromagnétique. Pour obtenir une vraie absorption (irréversibilité), il faut une dissipation. Celle ci peut être obtenue par les collisions électron-ion (Bremsstrahlung inverse) conduisant au chauffage du plasma, ou bien par des effets non linéaires générant des électrons suprathermiques.

L'utilisation d'une onde laser en polarisation $p$ et en incidence oblique $[19,21$ 23], dont une composante du champ électrique est normale à la cible, favorise la conversion de l'énergie laser en électrons suprathermiques. Sur le plan théorique, deux 
mécanismes ont êté identifiés. Le premier est l'absorption résonnante (à la densité critique) qui est contrôlée par la longueur du gradient de densité "L". Il a fait l'objet de nombreuses études en impulsions longues [24, 25]. En impulsions courtes (gradient raide) et haut flux, les mécanismes classiques de saturation de la résonance laissent place au deferlement (croisement des trajectoires électronique des électrons qui oscillent avec des amplitudes variant rapidement avec la position). En gradient ultracourt $\mathrm{L}<<\lambda$, l'absorption résonnante disparaît au profit de l'effet Brunel [14]. Dans ce mécanisme, les électrons situés à la surface du solide sont directement extraits par le champ électrique de l'onde incidente, puis précipités dans le solide lors de l'inversion du champ. Les vitesses de pénétration sont de l'ordre de la vitesse d'oscillation d'un électron dans le champ de onde incidente.

\subsubsection{Expérience réelle}

Sur le plan expérimental la situation est bien plus complexe. Le seuil de dommage d'un matériau solide est de l'ordre de $10^{9} \mathrm{~W} / \mathrm{cm}^{2}$, soit sept ordres de grandeur inférieur au flux crête. Le principal problème est donc posé par le contraste entre l'impulsion principale du laser et ce qui la précède (pré-impulsion ou piédestal). Dans le cas d'un laser de type Titane-Saphir, ce sont les défauts de la recompression de l'impulsion après amplification qui viendront limiter le contraste. Dans le cas présent de l'amplification directe par colorant, ce sera l'ASE (Émission Spontanée Amplifiée).

Schématiquement on peut distinguer deux modes d'action de la pré-impulsion.

Dans le premier mode, un plasma étendu est formé en avant de la cible solide. L'impulsion principale peut être absorbée efficacement à la densité critique mais la zone dense est alors faiblement chauffée. L'émission $\mathrm{X}$ thermique n'est alors pas favorisée par la faible densité de la région chauffée directement. Dans ces conditions, l'augmentation de l'émission $X$ observée [26] est due à une augmentation très importante du temps de refroidissement du plasma et donc de la durée d'émission $X$.

Dans le second mode, c'est l'état de surface de la cible qui est modifié par la formation d'une rugosité sous l'influence d'instabilités de surface, le gradient de densité restant très raide à l'interface solide-vide. L'augmentation de l'absorption pourrait s'expliquer par un couplage de l'énergie laser incidente avec les modes de plasmons de surface [27]. De même il a aussi été montré que des surfaces de cible initialement rugueuses favorisent l'absorption de l'énergie laser [28]. Dans ces conditions l'incidence oblique n'est plus indispensable pour obtenir une bonne absorption. Le chauffage direct du solide subsiste et une émission X de courte durée est alors possible.

Nos précédentes expériences [29] ont montré que l'émission $X$ est fonction du niveau de l'ASE. L'étude systématique de la production $\mathrm{X}$ en fonction du flux de l'ASE a montré que pour une fluence critique de $0.75 \mathrm{~J} / \mathrm{cm}^{2}$ le couplage laser cible est optimal [30]. Pour ce flux le gradient de densité électronique à la densité critique reste petit devant la longueur d'onde du laser et la diffusion du faisceau incident à grand angle est très importante. Cette diffusion étant associée à la rugosité, ces observations nous permettent de conclure que nous sommes situé dans le second mode d'action de la préimpulsion. Dans l'expérience présente, nous nous sommes donc placé à l'optimum de la production des $\mathrm{X}$

\section{MESURE ET INTERPRÉTATION DU RAYONNEMENT K}

\subsection{Dispositif expérimental}

L'objectif de l'expérience est de quantifier l'émission X du plasma et de déterminer la fonction de distribution de électrons rapides responsables de l'émission $\mathrm{K} \alpha$.

L'impulsion laser (100 fs FWHM, $1.5 \mathrm{~mJ}, 620 \mathrm{~nm}$ ) est obtenue à partir d'un oscillateur fonctionnant en mode bloqué, du type CPM ("colliding pulse modelocked"), et suivi par une série d'amplificateurs à colorants pompés à $10 \mathrm{~Hz}$ par un laser Nd:YAG. Une lentille ouverte à f/8 et positionnée à $7^{\circ}$ par rapport à l'incidence normale, permet d'obtenir des flux sur cible de l'ordre de $310^{16} \mathrm{~W} / \mathrm{cm}^{2}$ avec une tache focale de 7 microns (FWHM), proche de la limite de diffraction. 
La profondeur de pénétration des électrons dans la cible dépend de leur énergie. Compte tenue de celle prévue par la théorie $(10 \mathrm{keV})$ la profondeur de pénétration est de l'ordre du micron. La mesure de celle-ci a été réalisée à partir de l'émission X de cibles bicouches constituées de films d'aluminium de différentes épaisseurs déposés sur des substrats de $\mathrm{SiO}_{2}$ et $\mathrm{CaF}_{2}$.

Les raies d'émission $\mathrm{X}$ des deux matériaux constituant la cible sont collectées, simultanément, par un double spectrographe du type VonHamos qui est constitué de deux cristaux courbes placés côte à côte . Ces cristaux de PET (pentaerythritol, $2 \mathrm{~d}=$ $8.742 \AA$ ) ou de $\mathrm{LiF}$ (fluorure de calcium, $2 \mathrm{~d}=4.027 \AA$ ) ont un rayon de courbure de 10 $\mathrm{cm}$ et focalisent le rayonnement X sur le détecteur (film Kodak SB392) .

\subsection{Analyse sommaire des résultats expérimentaux .}

Les spectres $\mathrm{X}$ observés montrent la contribution d'émissions thermique et suprathermique. Leur localisation spatiale est déduite des variations d'intensité mesurées en fonction de l'épaisseur de la couche superficielle d'aluminium.

La raie d'émission de résonance $1 \mathrm{~s}^{2}-1 \mathrm{~s} 2 \mathrm{p}$ et ses satellites di-électroniques (composante thermique) sont émis jusqu'à une profondeur de $2500 \AA$ dans la cible [39] et correspondent au plasma thermique d'aluminium dont la température de l'ordre de $500 \mathrm{eV}$.

Sur une plus grande profondeur (10 $000 \AA$ ) nous observons les raies $K_{\alpha}$ de l'aluminium "froid" ( transitions des ions $\mathrm{Al}^{0+}$ et $\mathrm{Al}^{4+}$ qui se recouvrent) et l'émission $\mathrm{K}_{\alpha}$ décalée en longueur d'onde des ions $\left(\mathrm{Al}^{5+}\right.$ à $\mathrm{Al}^{9+}$ ). Ces dernières raies sont la signature d'un plasma dense chauffé à une température de l'ordre de $50 \mathrm{eV}$. Ce chauffage sera attribué au dépôt d'énergie par les électrons rapides.

La pénétration d'électrons monoénergétiques de $16 \mathrm{keV}$ pourrait rendre compte de la profondeur de la zone émissive des $K_{\alpha}[40-41]$. Comme nous le verrons plus loin, la fonction de distribution en énergie des électrons est bien sûr plus élargie. [7].

\subsection{Analyse des résultats à l'aide de la simulation numérique de la} pénétration des électrons dans le solide.

Pour aller plus loin dans l'interprétation des variations de l'intensité des raies en fonction de l'épaisseur de la couche superficielle, nous avons analysé nos résultats expérimentaux par comparaison avec les résultats d'un code de simulation numérique de la pénétration des électrons dans le solide. Par une méthode de Monte-Carlo, ce code détermine les trajectoires 3D d'un électron unique (pas de champ électrique), interagissant avec la cible par collisions élastiques (section efficace de Rutherford) et par collisions inélastiques (ralentissement de Bethe). En pondérant les résultats obtenus pour différentes énergies d'électrons, nous pouvons prédire les caractéristiques de pénétration d'une fonction de distribution plus complexe. Les efficacités de fluorescence, incluant le rendement Auger, sont utilisées pour déterminer le profil de l'émission $\mathrm{K}_{\alpha}$ en fonction de la profondeur de la cible. La profondeur optique du point d'émission à la surface de la cible est prise en compte. L'influence de ces effets d'opacité est particulièrement important pour les cibles $\mathrm{Al} / \mathrm{SiO}_{2}$ car le seuil de photoabsorption de l'aluminium se situe juste en dessous de l'énergie de la raie $\mathbf{K}_{\alpha}$ du silicium.

Pour diminuer l'importance des variations liées aux conditions expérimentales il est utile de s'intéresser au rapport d'intensité entre la raie $\mathrm{K}_{\alpha}$ du substrat et celle de la couche superficielle d'aluminium. La fonction de distribution des électrons est déterminé à partir des variations du rapport des rạies en fonction de l'épaisseur du film d'aluminium. Les résultats pour le substrat de calcium sont présentés sur la figure 1. Ce substrat est particulièrement bien adapté à l'étude des électrons d'énergie supérieure à $10 \mathrm{kcV}$, et donc aux épaisseurs d'aluminium importante $(\approx 10000 \AA)$, car l'émission $K_{\alpha}$ est peu réabsorbée à travers l'aluminium. Nous pouvons observer que nos résultats expérimentaux sont bien représentés par des électrons monoénergétiques de $3,8,16,28$ et $50 \mathrm{keV}$ avec des contributions en énergie de $21 \%, 47 \%, 23 \%, 6.9 \%$ 
et $2.1 \%$. Pour apprécier l'importance de chacune des contributions nous avons ajouté les courbes $(\mathrm{a}, \mathrm{b}, \mathrm{c})$ de la figure 1 qui donnent les résultats successifs pour les groupes d'électrons monoénergétiques suivants: $[3,8,16,0,0]$ (a), $[3,8,16,28,0]$ : (b), $[3,8,16,28,50]:$ (c).

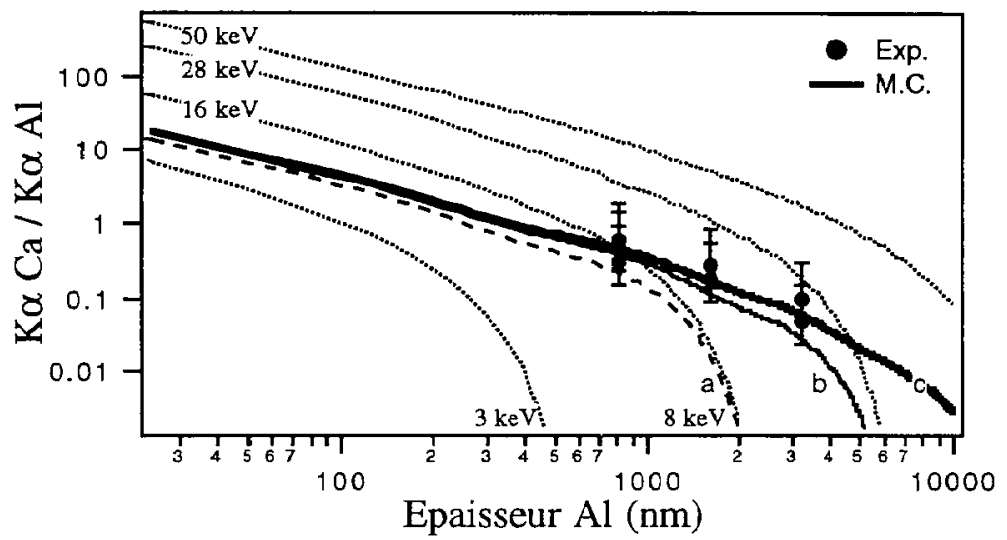

Figure 1 Rapport des raies $\mathrm{K}_{\alpha}$ "froide" du Ca et de $\mathrm{Al}$ en fonction de l'épaisseur d'aluminium. La courbe en trait plein représente le rapport calculé pour la fonction de distribution représentant le mieux les résultats expérimentaux. Les courbes $(\mathrm{a}, \mathrm{b}, \mathrm{c})$ montrent les différentes contributions d'électrons d'énergie au dessus de $16 \mathrm{keV}$ (voir texte). Les courbes en pointillés représentent les contributions des électrons monoénergétiques pour différentes énergies.

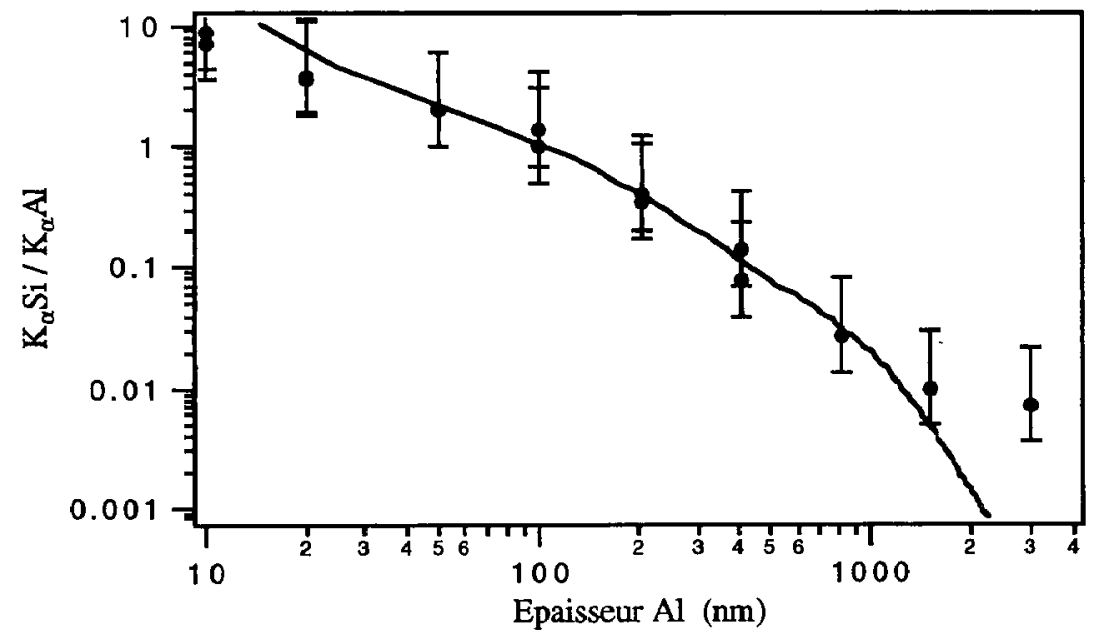

Figure 2: Rapport des raies $\mathrm{K}_{\alpha}$ de $\mathrm{Si}$ et $\mathrm{Al}$ en fonction de l'épaisseur de l'aluminium. La courbe en trait plein est le rapport calculé pour la même fonction de distribution que la figure $\mathrm{n}^{\circ} 1$. 
Les résultats obtenus avec la même distribution d'énergie, mais appliquée dans le cas du substrat de silice, sont présentés sur la figure 2. Il sont également comparés aux points expérimentaux. Nous pouvons remarquer l'excellent accord entre ces deux résultats (figure 1 et figure 2). La fonction de distribution ainsi déterminée est présentée sur la figure 3 . Pour valider notre analyse, nous avons changé les énergies du groupe d'électrons $[3,8,16,28,50] \mathrm{keV}$ en un autre groupe défini par $[3,6,10,20,40] \mathrm{keV}$. Les résultats sont présentés sur la figure 3 . Nous pouvons observer qu'ils sont en très bon accord avec ceux déterminés avec le premier groupe d'énergie. La pente de la fonction exponentielle déterminée à partir de ces points expérimentaux correspond à une température d'électrons chauds de $8.5 \mathrm{keV}$.

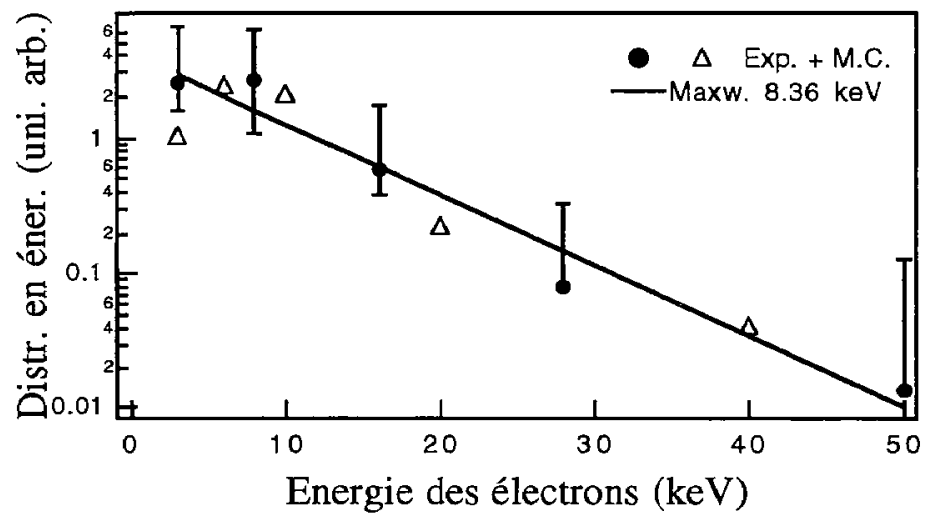

Figure 3: fonction de distribution en fonction de l'énergie des électrons rapides. Points: obtenu avec un groupe d'énergie de $[3,8,16,28,50] \mathrm{keV}$. Triangles: obtenus avec un groupe d'énergie de $[3,6,10,20,40] \mathrm{keV}$. Le trait plein est la pente de $8.5 \mathrm{keV}$ d'une fonction exponentielle qui représente le mieux les résultats expérimentaux.

\subsection{Flux des électrons rapides et du rayonnement $K_{\alpha}$}

La comparaison directe de l'intensité absolue de la raie $\mathrm{K}_{\alpha}\left(\mathbf{0 . 5} \pm \mathbf{0 , 2} \mathbf{J} / \mathbf{c m}^{2}\right)$ de l'aluminium froid avec les prédictions du code Monte-Carlo donne $\mathbf{1 2} \pm \mathbf{5} \%$ pour le rendement de conversion de l'énergie laser en électrons rapides.

La détermination des flux nécessite la mesure de la dimension de la zone émissive des raies $K_{\alpha}$. Nous avons utilisé la technique d'imagerie par couteau [51] qui donne une résolution spatiale meilleure que 2 microns. Pour s'affranchir du faible nombre de photons $\mathrm{X}$ à chaque tir, nous avons remplacé le film par un détecteur CCD refroidi $\left(-40^{\circ}\right)$, sensible au rayonnement $X$ et placé à $20^{\circ}$ de $1^{\prime}$ axe perpendiculaire à la cible. La résolution spectrale est alors de $100 \mathrm{eV}$ en utilisant la technique du comptage de photons [52], ce qui est suffisant pour séparer les raies $\mathrm{K}_{\alpha}$ de l'émission $\mathrm{He}_{\alpha} \mathrm{du}$ plasma thermique. Après déconvolution, nous obtenons une image de la zone émissive de la raie $K_{\alpha}$. Le diamètre ainsi déterminé est de 7 microns ce qui est légèrement superieur à la dimension de la tache focale du laser visible. Le flux d'électrons rapides correspondant, déterminé à partir de l'énergie du laser $(1.5 \mathrm{~mJ})$ et dẹ l'efficacité de conversion (12.5\%), est $197 \mathrm{~J} / \mathrm{cm}^{2}$, En prenant en compte cette valeur du flux il est possible de calculer l'élévation de température due au dépôt d'énergie des électrons dans le solide. Nous obtenons une température moyenne de la zone émissive de $30 \mathrm{eV}$, qui 
est en très bon accord avec les valeurs obtenues à partir des intensités relatives des raies $\mathrm{Al}^{0+}, \mathrm{Al}^{4+}$ et $\mathrm{Al}^{5+}$.

\section{GÉNÉRALISATION À D'AUTRES TRANSITIONS $K_{\alpha}$}

Connaissant la fonction de distribution des électrons rapides nous pouvons calculer la production de rayonnement $\mathrm{K}_{\alpha}$ pour différents matériaux. Les résultats en fonction du numéro atomique de la cible sont reportés sur la figure 4 . Elle présente le nombre de photons $\mathrm{K}_{\alpha}$ par stéradian calculés avec le code Monte-Carlo. Les effets de réabsorption ( ligne en pointillée sur la figure ) réduisent légèrement le nombre de photons $\mathrm{K}_{\alpha}$ pour les matériaux de faible $\mathrm{Z}$.

Pour valider ce calcul nous avons effectué des mesures sur des cibles de Calcium et de Fer. Les points expérimentaux correspondants sont reportés sur la figure 4 . Nous observons un excellent accord avec les prédictions théoriques ce qui confirme notre précédente analyse.

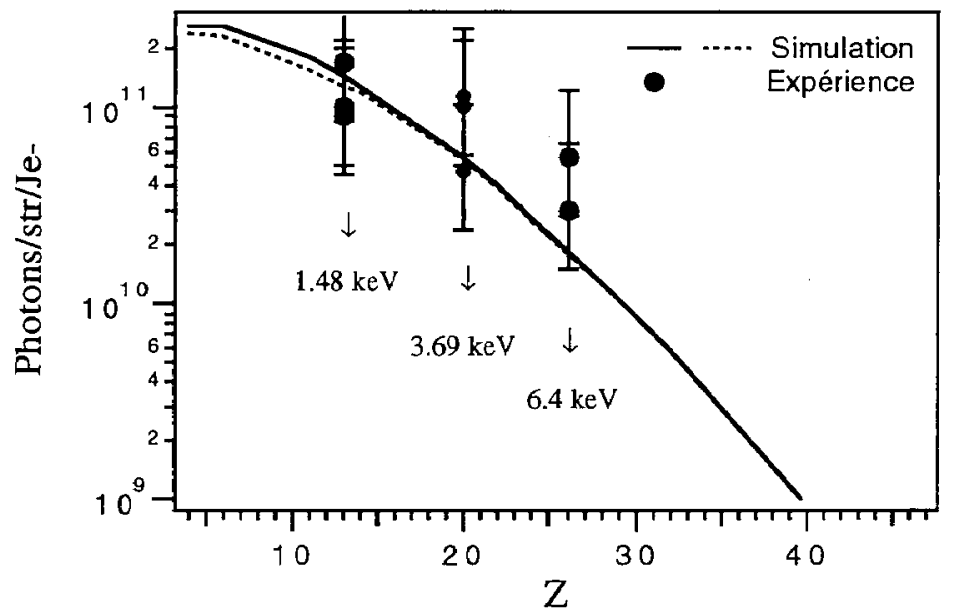

Figure 4 Nombre de photons $\mathrm{K}_{\alpha}$ émis par stéradian en fonction du matériau.

Trait plein: simulation Monte-Carlo sans réabsorption; trait pointillé: avec réabsorption. Point expérimentaux : substrat d'Aluminium, de Calcium, de Fer.

\section{CONCLUSION}

Nous avons caractérisé l'émission $\mathrm{K}_{\alpha}$ générée par l'interaction d'une impulsion laser ultracourte (100 fs) et intense $\left(310^{16} \mathrm{~W} / \mathrm{cm}^{2}\right)$ avec des cibles solides multicouches. L'intensité sur la raie $\mathrm{K}_{\alpha}$ est de $2.510^{7}$ photons/stéradian/impulsion pour de l'aluminium à $1.5 \mathrm{keV}$. Par des mesures absolues du rayonnement $\mathrm{K}_{\alpha}$ nous avons alors déterminé l'efficacité de conversion de l'énergie laser en électrons suprathermiques ( $12 \%$.) nous avons montré que la fonction de distribution de ces électrons est bien représentée par une fonction maxwellienne à $8.5 \mathrm{keV}$. Par ailleurs la dimension de la zone émissive $\mathrm{K}_{\alpha}$ est comparable à la tache focale du laser. Sachant que la durée de d'émission des électrons rapides n'est pas plus longue que la durée de l'impulsion laser, le courant d'électrons rapides est de $2.510^{5} \mathrm{MA} / \mathrm{cm}^{2}$. Nous avons démontré l'accordabilité de notre source de rayonnement $\mathrm{K}_{\alpha}$ pour des énergies comprises entre 1 et $10 \mathrm{KeV}$. 
Il faut noter l'importance essentielle de l'ASE dans l'optimisation de l'émission X. A ce jour, la compréhension théorique du rôle de l'ASE n'est pas complète. L'utilisation de lasers à très haut contraste, qui sont maintenant disponibles (Titane-Saphir), va nous permettre de maîtriser le couplage laser-cible en utilisant une pré-impulsion courte et parfaitement contrôlée. Dans ces conditions, la compréhension théorique deviendra moins difficile. Compte tenue des énergies laser prévues (300 millijoules), et en tablant sur une augmentation du rendement obtenu par une meilleure optimisation, un gain de trois ordres de grandeur sur les flux $\mathrm{X}$ pourrait être espéré dans les prochaines années.

\section{Références}

1. M.M. Murnaneet al., Science 251, 531 (1991).

2. D. Kuhlke, U. Herpes and D. Van der Linde, Appl. Phys. Letters, 50, 1785 (1987).

3. O.L. Landen, M. Campbell and M.D. Perry, Opt.Comm 63, 253 (1987).

4. H.M. Milchberg, R.R. Freeman and S.C. Davey, Phys. Rev. Letters 61, 2364 (1988).

5. D.G. Stearns et al., Phys. Rev. 37, 1684 (1988).

6. M.M Murnane, H.C. Kaypten and R.W. Falcone, Phys. Rev. Letters 62, 155 (1988).

7. P. Audebert et al., Europhys. Letters 19, 189 (1992).

8. J.C. Kieffer et al., Phys. Fluids B5, 2330 (1992).

9 H.M. Milchberg, I. Lyubomirsky and C.G.Durfee III, Phys. Rev. Letters 67, 2564(1991).

10 T. Teng, H.W. Huang and G.A. Olah, Biochemistry 26, 8066 (1987).

11 M.M. Murnane, H.C. Kapteyn and R.W. Falcone, IEEE Journal of

12. J. Denavit, Phys. Rev. Letters 69, 3052 (1992).

13. M. Rosen "Femtosecond to Nanosecond High-Intensity Lasers and Applications.", SPIE Proceedings 1229 (1990), p. 160.

14. F. Brunel, Phys. Rev. Letters 59, 52 (1987).

15. F. Brunel, Phys. Fluids 31, 2714 (1988).

16. G. Bonnaud et al., Laser and Part. Beams 9, 339 (1991).

17. E. Gamaly, Physics Fluids B5, 3765 (1993).

18. B.N. Chichkov, Y. Kato and M. Murakami, Phys. Rev. A46, 4512 (1992).

19. D.D. Meyerhofer et al., Physics Fluids B5, 2584 (1993).

20. A. Rousse et al. "Short Wavelength V: Physics with Intense laser pulses Technical Digest." Optical Society of America (1993) p. 60.

21. R. Fedosejevs et al., Appl. Physics B50, 79 (1990).

22. H. Chen et al., Phys. Rev. Letters 70, 3431 (1993).

23. U. Teubner et al., Phys. Rev. Letters 70, 794 (1993).

24. H. Maki and K. Niu, Jl. Phys. Soc. Japan 45, 269 (1978).

25. W.L. Kruer "The Physics of Laser Plasma Interactions." Vol. 73. (Redwood City, Addison-Wesley, 1988).

26 U. Teubner, G. Kühnle and F.P. Schäfer, Appl. Phys. B54, 493 (1992).

27 R. Dragila and E.G. Gamaly, Phys. Rev. 44A, 6828 (1991); E.G. Gamaly, Phys. Rev. 48E, 516 (1993).

28 S.P. Gordon et al. "Short Wavelength V: Physics with Intense laser pulses Technical Digest." Optical Society of America (1993) p. 117.

29 R. Benattar et al., Optics Comm. 88, 376 (1992).

30 P. Audebert et al. . AIP Conf. Proc. 257 (Portland, 1991), p. 58.

31 E.G. Chowanietz, D.H. Lumb and A.A. Wells, SPIE Proceedings 587 